\title{
Theoretical utmost performance of (100) mid-wave HgCdTe photodetectors
}

\author{
P. Martyniuk ${ }^{1}$ - W. Gawron' ${ }^{1}$ P. Madejczyk ${ }^{1}$ • \\ M. Kopytko ${ }^{1} \cdot$ K. Grodecki ${ }^{1}$. E. Gomułka ${ }^{1}$
}

Received: 29 August 2016/ Accepted: 22 November 2016/Published online: 29 December 2016

(C) The Author(s) 2016. This article is published with open access at Springerlink.com

\begin{abstract}
HgCdTe}$ detectors designed to detect mid-wavelength $(3-5 \mu \mathrm{m})$ infrared radiation must be cooled to reach the required performance. The cooling requirement makes the sensor system both expensive and bulky and the fundamental goal is to reach higher operating temperature condition preserving near background limited performance with high detectivity and high speed response at the same time. In order to reach higher operating temperature condition the thermal generation rate must to be suppressed under the photon generation rate. Except Auger 7 generation-recombination process, $p$-type $\mathrm{HgCdTe}$ is mostly limited by technology dependent Shockley-Read-Hall generationrecombination mechanism. One of the ways to reduce the trap density is a growth of the (100) $\mathrm{HgCdTe}$ on GaAs substrates. That orientation allows reaching lower carrier concentration $\sim 5 \times 10^{14} \mathrm{~cm}^{-3}$ in comparison to the commonly used (111) orientation $\sim 5 \times 10^{15} \mathrm{~cm}^{-3}$ in mid-wavelength infrared range. In addition, it was presented that Shockley-Read-Hall traps density could be reduced to the level of $\sim 4.4 \times 10^{8} \mathrm{~cm}^{-3}$. The theoretical simulations related to the utmost performance of the (100) $\mathrm{HgCdTe}$ Auger suppressed structures are presented. Dark current is reported to be reduced by more than one order of magnitude within the range $\sim 6 \times 10^{-2}-3 \times 10^{-3} \mathrm{~A} / \mathrm{cm}^{2}$. Detectivity increases within range $\sim 3-12 \times 10^{11} \mathrm{~cm} \mathrm{~Hz}^{1 / 2} / \mathrm{W}$ (wavelength $\sim 5 \mu \mathrm{m}$ ) at temperature $200 \mathrm{~K}$ and voltage $200 \mathrm{mV}$.
\end{abstract}

Keywords (100) HgCdTe orientation · MWIR · HOT

This article is part of the Topical Collection on Numerical Simulation of Optoelectronic Devices 2016.

Guest edited by Yuh-Renn Wu, Weida Hu, Slawomir Sujecki, Silvano Donati, Matthias Auf der Maur and Mohamed Swillam.

P. Martyniuk

piotr.martyniuk@wat.edu.pl

1 Institute of Applied Physics, Military University of Technology, 2 Kaliskiego Str., 00-908 Warsaw, Poland 


\section{Introduction}

Without optical immersion (GaAs substrate-converted into immersion lens) mid-wavelength infrared radiation (MWIR) HgCdTe photovoltaic detectors are reported to exhibit nearly background limited performance (BLIP) with performance close to the generationrecombination (GR) limit, but well designed optically immersed devices approach BLIP condition when thermoelectrically cooled with 2-stage Peltier coolers (Piotrowski and Rogalski 2007; Rogalski 2011). As substrates, epiready GaAs wafers of different orientation and interdiffused multilayer process (IMP) are used in $\mathrm{HgCdTe}$ growth by MOCVD in our laboratory (Irvine 1992). Epiready (100) GaAs substrates exhibit $\sim 14.6 \%$ lattice mismatch with CdTe buffer layer used in our structures. That mismatch allows to grow both (100) and (111) HgCdTe orientations. That mostly depends on substrate disorientation, nucleation conditions and growth temperature. In addition, the monolithic GaAs optical immersion results in significant improvement in detectivity by $\sim n^{2}$, where $n$ stands for GaAs refractive index giving flexibility in detector's optimization in terms of time constant $\left(\tau_{s}\right)$ and detectivity $\left(D^{*}\right)$ (Piotrowski and Rogalski 2004).

Figure 1a, b shows the difference in surface morphology between two analyzed $\mathrm{HgCdTe}$ orientations. As it is presented in Fig. 1, the (100) $\mathrm{HgCdTe}$ orientation tends to be almost mirror-smooth. In addition (100) $\mathrm{HgCdTe}$ epilayers have higher $p$-type Arsenic (As) doping efficiency and is an attractive plane for fabrication of the abrupt heterojunctions.

Even though the (100) surface morphology is superior to the (111), the (100) orientation is characterized by pyramid-shaped macrodefects known as hillocks shown in both surface and cleavage presented in Fig. 2a, b. Epilayers with hillocks are practically useless for device fabrication. Origin of hillocks is not fully understood at present. They were found to be expanded defects created during CdTe buffer growth and further $\mathrm{HgCdTe}$ deposition only enlarges them. Several approaches to prevent hillocks creation have been tried: zinc nucleation layer, different $\mathrm{Cd} / \mathrm{Te}$ ratios, different substrates orientations have been used, but we were not able to grow hillocks-free layer in controllable way on GaAs substrates. According to literature, only Selex Galileo reported on suppression of hillocks density below $5 \mathrm{~cm}^{-2}$ in the (100) $\mathrm{HgCdTe}$ grown on GaAs (Maxey et al. 2000, 2006). Therefore, most of our reported MWIR HgCdTe $\mathrm{N}^{+} \mathrm{pP}^{+} \mathrm{n}^{+}$devices are based on (111) HgCdTe layers (Madejczyk et al. 2009a, b, 2013).

(a)

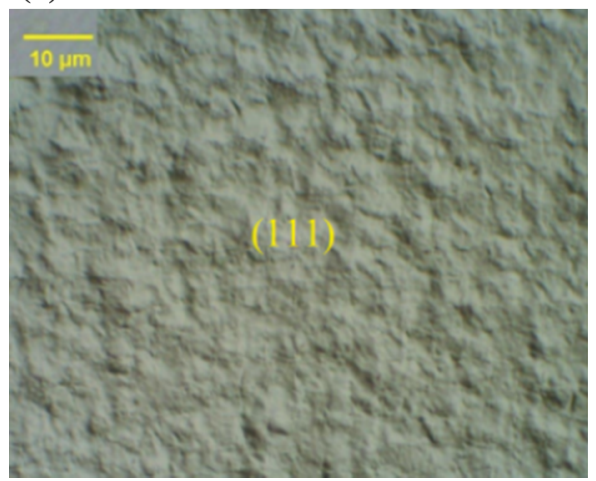

(b)

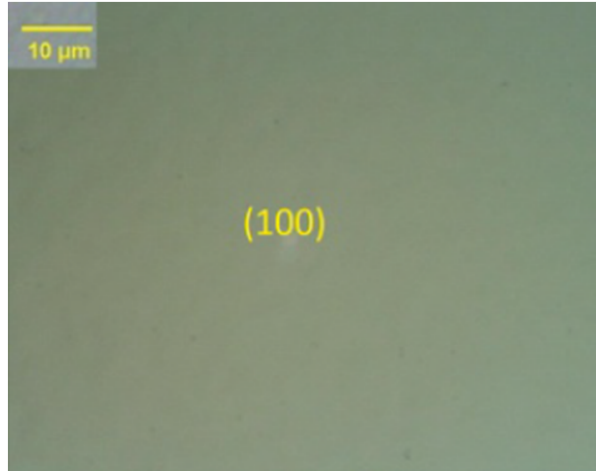

Fig. 1 Surface morphology of (111) (a) and (100) (b) HgCdTe layers 
(a)

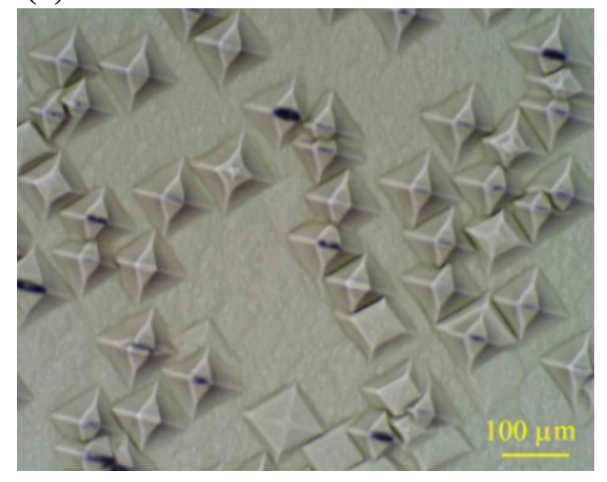

(b)

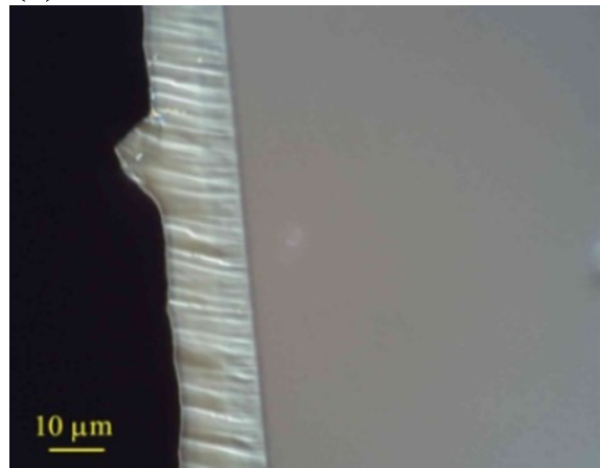

Fig. 2 Hillocks on (100) HgCdTe layers: surface (a); cleavage (b)

High-quality MWIR detectors require HgCdTe layers with low dislocation density. The (100) $\mathrm{HgCdTe}$ orientation allows to reduce $p$-type doping to the level of $\sim 5 \times 10^{14} \mathrm{~cm}^{-3}$ in analyzed MWIR range. In addition Shockley-Read-Hall (SRH) traps density could be reduced to the level of $\sim 4.4 \times 10^{8} \mathrm{~cm}^{-3}$. According to Maxey et al. in the structures with (100) orientation the SRH trap concentration follows following equation: $N_{\text {Trap }}=3 \times 10^{-11} \mathrm{~N}_{\mathrm{A}}^{1.44}$ which for active layer doping $N_{A}=5 \times 10^{14} \mathrm{~cm}^{-3}$ results in $N_{\text {Trap }}=4.4 \times 10^{8} \mathrm{~cm}^{-3}$ assumed in simulations (Maxey et al. 2006). Previously Maxey et al. reported on slightly different relation: $N_{\text {Trap }}=3 \times 10^{-11} \mathrm{~N}_{\mathrm{A}}^{1.353}$ and measured carrier concentrations $N_{A} \leq 8 \times 10^{15} \mathrm{~cm}^{-3}$ and trap density $N_{\text {Trap }} \leq 9 \times 10^{10} \mathrm{~cm}^{-3}$ being independent of Cadmium (Cd) composition, $x_{C d}$ (Maxey et al. 2000). The $77 \mathrm{~K}$ carrier concentration for the Cd composition for two analyzed orientations (111) and (100) is presented in Fig. 3. The carrier concentrations assumed in calculations are fully confirmed within the range composition corresponding to the MWIR range reached in our MOCVD machine. In this paper we present the theoretical simulations related to the utmost performance: detectivity and time constant of the (100) $\mathrm{HgCdTe}$ MWIR, $\mathrm{N}^{+} \mathrm{pP}^{+} \mathrm{n}^{+}$multilayer structures grown on GaAs substrates.

Fig. 3 Measured $77 \mathrm{~K}$ carrier concentration for analyzed (100) and (111) orientations

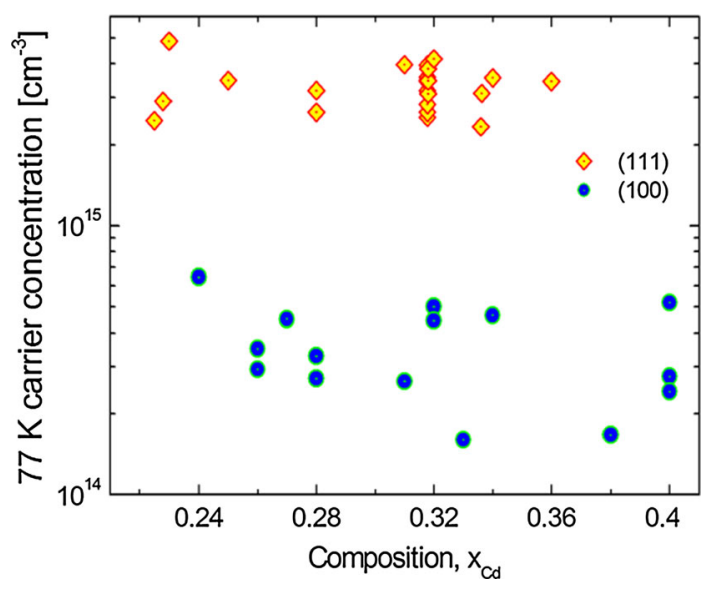




\section{Simulation procedure and results}

The detailed description of the (111) orientation HgCdTe MWIR detector structure grown on GaAs substrate was described in our previous paper (Martyniuk et al. 2014). In our new approach for (111) orientation MWIR HgCdTe $p$-type doping, $N_{A}=5 \times 10^{15} \mathrm{~cm}^{-3}$ and trap density, $N_{\text {Trap }}=2.3 \times 10^{13} \mathrm{~cm}^{-3}$ in active layer were assumed while for (100) orientation the doping was reduced to correspond to the level presented in Fig. 3, i.e. $N_{A}=5 \times 10^{14} \mathrm{~cm}^{-3} \quad\left(x_{C d} \sim 0.26\right)$ and according to the Maxey's expression: $N_{\text {Trap }}=3 \times 10^{-11} \mathrm{~N}_{\mathrm{A}}^{1.353}$ trap level density to $N_{\text {Trap }}=4.4 \times 10^{8} \mathrm{~cm}^{-3}$. In both cases (111) and (100) orientation $\mathrm{HgCdTe}$ detectors we used well known architecture $\mathrm{N}^{+} \mathrm{pP}^{+} \mathrm{n}^{+}$ for non-equilibrium condition shown in Fig. 4 assuming proper grading at the heterojunctions: $\mathrm{N}^{+}-\mathrm{p}, \mathrm{p}-\mathrm{P}^{+}$and $\mathrm{P}^{+}-\mathrm{n}^{+}$(Ashley and Elliott 1985). The $x_{C d}$ composition and doping gradients were assumed to have linear dependence on the thickness of the particular layers.

The standard (111) MWIR HgCdTe structure where narrow-gap absorber is inserted between wider carrier contacts with an absorber average composition, $x_{C d} \sim 0.26$, thickness, $d=6 \mu \mathrm{m}$, and $p$-type doping $N_{A} \approx 5 \times 10^{15} \mathrm{~cm}^{-3}$ is shown in Fig. 4. The main layers are interfaced with thin graded gap and doping level transition layers formed by IMP technique during the growth procedure, e.g. layer 2 (thickness $0.7 \mu \mathrm{m}$ ) $x_{C d}$ composition lowers within the range $x_{C d}=0.34-0.3$, while doping gradient $N_{D}=50-5 \times 10^{16} \mathrm{~cm}^{-3}$. The device presented in this paper was fabricated in the joint laboratory run by VIGO Systems and the Military University of Technology (MUT). The HgCdTe epiready layers were grown on semi-insulating (100) GaA substrates in a horizontal MOCVD AIX 200 reactor. It was assumed that the device was illuminated through the $\mathrm{N}^{+}$layer acting as an infrared transmitting window.

Theoretical simulations of both (111) and (100) orientations HgCdTe heterostructures have been performed by numerical solving of Poisson's and the electron/hole current continuity equations by the Newton-Richardson method. APSYS platform (by Crosslight Inc.) was implemented in our simulation procedure. The proper equations describing the drift-diffusion model are presented in detail in the APSYS manual (APSYS 2011). Ohmic contacts were modeled as Dirichlet boundary conditions where both electron $\left(E_{f n}\right)$ and hole $\left(E_{f p}\right)$ quasi-Fermi levels are equal and assumed to be at the voltage of electrode following the relation: $E_{f n}=E_{f p}=V$. The used model assumes electrical and optical properties to include the influence of radiative (RAD), Auger (AUG), SRH GR at any mesh point within the device and band-to-band (BTB) as well as trap assisted (TAT) tunnelling mechanisms

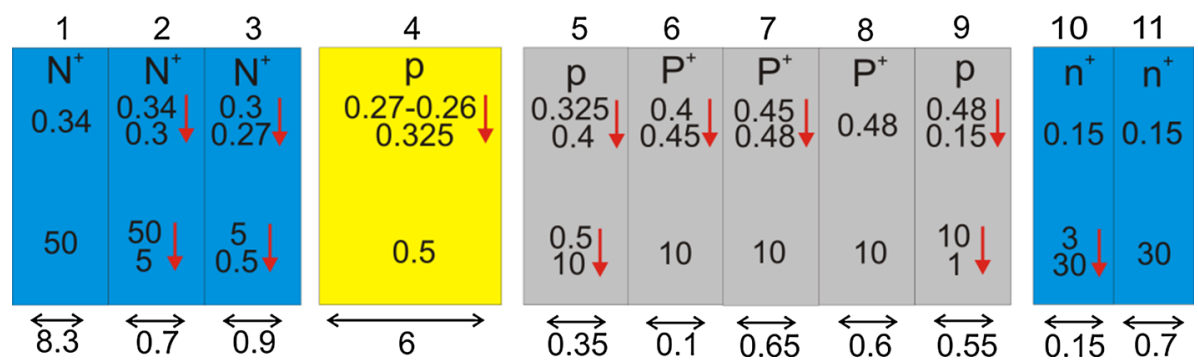

Fig. 4 Simulated (111) $\mathrm{N}^{+} \mathrm{pP}^{+} \mathrm{n}^{+} \mathrm{HgCdTe}$ heterostructure. The data in the rows: layer number, type of doping, $x_{C d}$ composition grading, doping grading $\times 10^{16} \mathrm{~cm}^{-3}$, and thickness of the layers in $\mu \mathrm{m}$ are marked. Red arrow presents composition and doping grading. (Color figure online) 
at the $\mathrm{N}^{+}-\mathrm{p}\left(\mathrm{N}^{+}\right.$contact- $p$-type absorber) heterojunction. AUG recombination mechanisms using Casselman et al. approximation of parabolic bands and non-degenerate statistics was implemented (Casselman and Petersen 1980). Energy bandgap was calculated after the paper by Hansen et al. (1982). The zero voltage electron mobility was taken from the formula based on Scott's paper, where the hole mobility was assumed as 0.01 of the electron mobility (Scott 1972). Intrinsic concentration's composition and temperature dependence was calculated based on the Hansen et al. model (Hansen and Schmidt 1983). For the TAT simulation the Hurkx et al. model, which is similar to the SRH GR formula, was implemented (Hurkx et al. 1992). The absorption was assumed in active layer region and absorption coefficient was estimated according to Kane model including its composition, doping and temperature dependences (e.g. $\alpha=5470 \mathrm{~cm}^{-1}, \lambda=5 \mu \mathrm{m}, T=200 \mathrm{~K}$ ). The TAT mechanism was found to be important for fitting to experimental results for (111) $\mathrm{HgCdTe}$ structure presented in Fig. $5 \mathrm{a}$ by assuming a trap concentration, $N_{\text {Trap }}$ $\sim 10^{13} \mathrm{~cm}^{-3}$, and trap energy related to the conduction band according to the relation: $E_{\text {Trap }}=0.33 \times E_{g}$. Simulation of time constant was performed using $\mathrm{Li}$ et al. model ( $\mathrm{Li}$ and Dutton 1991). Proper doping grading were introduced to prevent form discontinuities in energy band profiles between contact-absorber $\left(\mathrm{N}^{+}-\mathrm{p}\right)$, absorber-barrier $\left(\mathrm{p}-\mathrm{P}^{+}\right)$and finally barrier-contact $\left(\mathrm{P}^{+}-\mathrm{n}^{+}\right)$heterojunctions. The detailed parameters taken in modelling of MWIR (111) and (100) orientations HgCdTe heterostructures are presented in Table 1.

Measured and simulated $J_{D A R K}$ versus voltage for both (111) and (100) orientations are presented in Fig. 5. Active layer doping reduction to the level presented in Fig. 3 suppresses both BTB and TAT mechanisms. Slight Auger suppression is seen above $290 \mathrm{~K}$ for (100) orientation structure (Fig. 5b). The proper correspondence was reached for simulated and measured values at $T=200 \mathrm{~K}$ for (111) $\mathrm{HgCdTe} \mathrm{N}^{+} \mathrm{pP}^{+} \mathrm{n}^{+}$heterostructure. For higher voltages dark current is mostly dependent on TAT mechanism at the $\mathrm{N}^{+}-\pi$ (contact layer-absorber) heterojunction for (111) orientation. At lower temperatures Auger suppression is barely visible being covered by TAT and SRH due to the fact that average trap density for (100) orientation was estimated to be within the range $\sim 10^{8} \mathrm{~cm}^{-3}$. Extraction coefficient was calculated for $300 \mathrm{~K}$ assuming $=1.1$. Simulations for (100) orientation were performed for active layer doping $N_{A}=5 \times 10^{14} \mathrm{~cm}^{-3}$ and SRH trap density, $N_{\text {Trap }}=3 \times 10^{-13} \mathrm{~N}_{\mathrm{A}}^{1.44}=4.4 \times 10^{8} \mathrm{~cm}^{-3} . J_{\text {DARK }}$ suppression for $200 \mathrm{mV}$ was found to
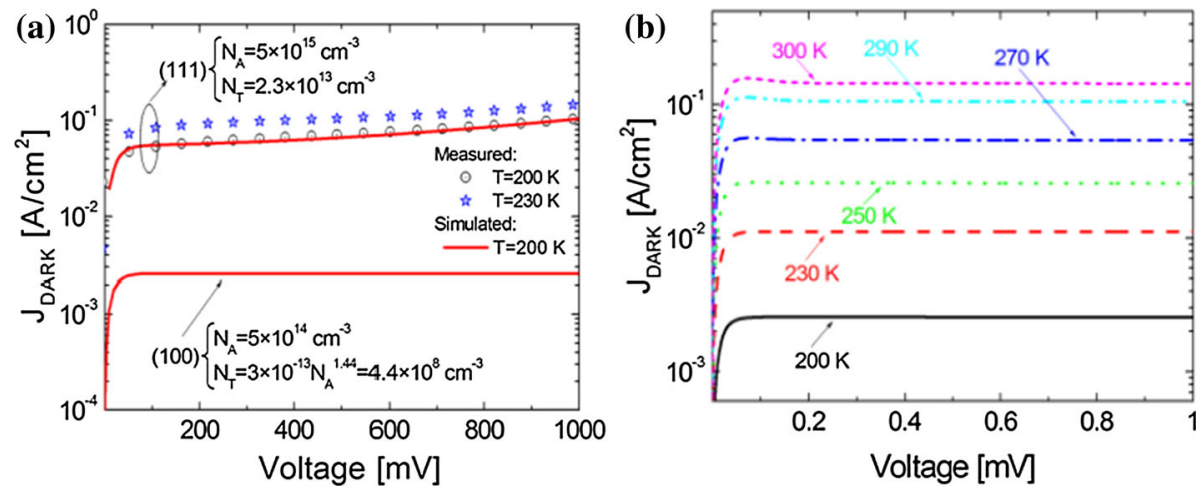

Fig. 5 Theoretically simulated and measured dark current density versus voltage for (111) orientation grown on GaAs-absorber doping, $N_{A}=5 \times 10^{15} \mathrm{~cm}^{-3}$; trap density, $N_{\text {Trap }}=2.3 \times 10^{13} \mathrm{~cm}^{-3}$ and (100) orientation absorber doping, $N_{A}=5 \times 10^{14} \mathrm{~cm}^{-3}$; trap density, $N_{\text {Trap }}=4.4 \times 10^{8} \mathrm{~cm}^{-3}$ (a). $J_{\text {DARK }}$ versus voltage for selected temperatures, $T=200-300 \mathrm{~K}(\mathbf{b})$ 
Table 1 Parameters taken in modelling of MWIR (111)* and (100)** orientations $\mathrm{HgCdTe}$ heterostructures

\begin{tabular}{lllll}
\hline & Contact layer & Active layer & Barrier layer & Contact layer \\
\hline Doping, $N_{A} ; N_{D}\left(\mathrm{~cm}^{-3}\right)$ & $50 \rightarrow 0.5^{* /}$ & $5 \times 10^{15 * ;}$ & $0.5^{* / 0.05^{* *} \rightarrow 10}$ & $3 \rightarrow 30 \times 10^{16}$ \\
& $0.05^{* *} \times 10^{16}$ & $5 \times 10^{14^{* *}}$ & $\rightarrow 1 \times 10^{16}$ & \\
Doping Gauss tail, $d x(\mu \mathrm{m})$ & 0.05 & & \\
Composition, $x, y$ & $0.34 \rightarrow 0.27$ & $0.27 \rightarrow 0.325$ & $0.15 \rightarrow 0.4$ & 0.15 \\
Geometry, $d \mu \mathrm{m})$ & 9.9 & 6 & 2.25 & 0.85 \\
Electrical area, $A\left(\mu \mathrm{m}^{2}\right)$ & $100 \times 100$ & & \\
Overlap matrix, $F_{1} F_{2}$ & 0.2 & & \\
Trap energy level, $E_{\text {Trap }}$ & $0.33 \times \mathrm{E}_{\mathrm{g}}$ & & \\
Trap concentration, & $2.3 \times 10^{13 *} ;$ & & \\
$\quad N_{\text {Trap }}\left(\mathrm{cm}{ }^{-3}\right)$ & $4.4 \times 10^{8 * *}$ & & \\
SRH & & & \\
$\sigma_{\mathrm{n}}\left(\mathrm{cm}^{-2}\right)$ & $5 \times 10^{-15}$ & & \\
$\sigma_{\mathrm{p}}\left(\mathrm{cm}^{-2}\right)$ & $5 \times 10^{-15}$ & & \\
Incident power density, & 500 & & \\
$\quad \Phi\left(\mathrm{W} / \mathrm{m}^{2}\right)$ & & & \\
\hline
\end{tabular}

be more one order of magnitude in the region where TAT mechanism is playing a decisive role for (111) orientation.

Detectivity was also calculated. In order to assess $D^{*}$, the noise current was simulated using the following expression to include both the thermal Johnson-Nyquist noise and electrical shot noise contributions:

$$
i_{n}(V)=\sqrt{\left(4 k_{B} T / R A+2 q J_{D A R K}\right) A},
$$

where $A$ is the area of the detector $\left(100 \times 100 \mu \mathrm{m}^{2}\right), R A$ is the dynamic resistance area product, $J_{D A R K}$ is the dark current density, and $k_{B}$ is the Boltzmann constant. Detectivity is defined by the following expressions to include the effect of the GaAs immersion lens $(n$ GaAs refractive index):

$$
D^{*}=\frac{R_{i}}{i_{n}(V)} n^{2} \sqrt{A} .
$$

The structure with (100) orientation and integrated GaAs immersion lens reaches $\sim 10^{12} \mathrm{~cm} \mathrm{~Hz}^{1 / 2} / \mathrm{W}(T=200 \mathrm{~K})$ being one order magnitude higher than BLIP detectivity $\sim 10^{11} \mathrm{~cm} \mathrm{~Hz}^{1 / 2} / \mathrm{W}$ at $\lambda \sim 5 \mu \mathrm{m}$ and reported previously for structures with (111) orientation presented in Fig. 6 .

Time response was simulated versus voltage (Fig. 7a) and temperature for $V=250 \mathrm{mV}$ (Fig. 7b). For the (100) orientation time response, $\tau_{s}$ reaches $\sim 4100-1100$ ps for voltage range 50-400 $\mathrm{mV}$ being nearly two times lower in comparison to the (111) orientation for $V=400 \mathrm{mV}$. Series resistance was assumed to be within range 190-510 $\Omega$. Experimental data presented in Fig. 7a was plotted for (111) orientation. 
Fig. 6 Theoretically simulated and measured detectivity versus wavelength for (111) orientation grown on GaAs-absorber doping, $N_{A}=5 \times 10^{15} \mathrm{~cm}^{-3}$; trap density, $N_{\text {Trap }}=2.3 \times 10^{13}$ $\mathrm{cm}^{-3}$ and (100) orientation absorber doping, $N_{A}=5 \times 10^{14}$ $\mathrm{cm}^{-3}$; trap density, $N_{\text {Trap }}=4.4 \times 10^{8} \mathrm{~cm}^{-3}$
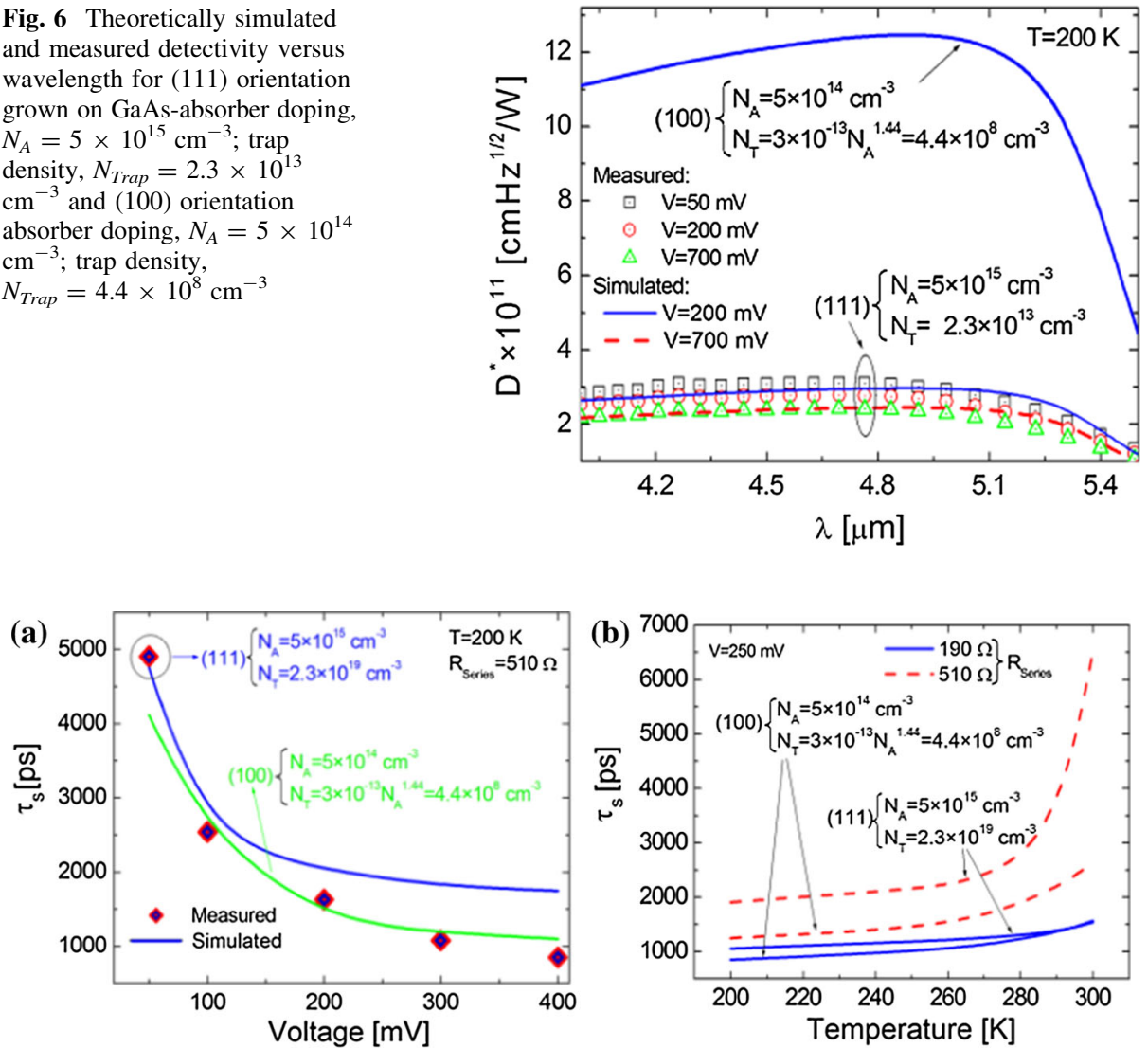

Fig. 7 Measured and theoretically simulated time response versus voltage (a) and temperature (b) for (111) orientation grown on GaAs-absorber doping, $N_{A}=5 \times 10^{15} \mathrm{~cm}^{-3}$; trap density, $N_{\text {Trap }}=2.3 \times 10^{13} \mathrm{~cm}^{-3}$ and (100) orientation absorber doping, $N_{A}=5 \times 10^{14} \mathrm{~cm}^{-3}$; trap density, $N_{\text {Trap }}=4.4 \times 10^{8} \mathrm{~cm}^{-3}$. $R_{\text {Series }}=190$ and $510 \Omega$

\section{Conclusions}

Theoretical utmost performance of the (100) $\mathrm{HgCdTe}$ grown on GaAs substrate MWIR photodetector was presented. It is predicted that trap density could be reduced to $\sim 4.4 \times 10^{8} \mathrm{~cm}^{-3}$ assuming active layer doping $\sim 5 \times 10^{14} \mathrm{~cm}^{-3}$. Those active layers parameters results in suppression of the dark current $\sim 6 \times 10^{-2}-3 \times 10^{-3} \mathrm{~A} / \mathrm{cm}^{2}$. Detectivity increases within range $\sim 3-12 \times 10^{11} \mathrm{~cm} \mathrm{~Hz}^{1 / 2} / \mathrm{W}$ at temperature $200 \mathrm{~K}$ and voltage $200 \mathrm{mV}$. Suppression of the trap density to the level of $\sim 4.4 \times 10^{8} \mathrm{~cm}^{-3}$ allows reaching better performance in frequency response $\sim 859 \mathrm{ps}$ corresponding to $200 \mathrm{~K}$ and $V=200 \mathrm{mV}, R_{\text {Series }}=190 \mathrm{~K}$.

Acknowledgements This paper has been completed with the financial support of the Polish National Science Centre, Projects: 2013/08/A/ST5/00773 and 2013/08/M/ST7/00913.

Open Access This article is distributed under the terms of the Creative Commons Attribution 4.0 International License (http://creativecommons.org/licenses/by/4.0/), which permits unrestricted use, distribution, 
and reproduction in any medium, provided you give appropriate credit to the original author(s) and the source, provide a link to the Creative Commons license, and indicate if changes were made.

\section{References}

APSYS Macro/User's Manual ver. 2011.: Crosslight Software, Inc. (2011)

Ashley, T., Elliott, C.T.: Non-equilibrium mode of operation for infrared detection. Electron. Lett. 21, 451-452 (1985)

Casselman, T.N., Petersen, P.E.: A comparison of the dominant Auger transitions in $p$-type (HgCd)Te. Solid State Commun. 33, 615-619 (1980)

Hansen, G.L., Schmidt, J.L.: Calculation of intrinsic carrier concentration in $\mathrm{Hg}_{1-\mathrm{x}} \mathrm{Cd}_{\mathrm{x}}$ Te. J. Appl. Phys. 54, 1639-1640 (1983)

Hansen, G.L., Schmidt, J.L., Casselman, T.N.: Energy gap versus alloy composition and temperature in $\mathrm{Hg}_{1-\mathrm{x}} \mathrm{Cd}_{\mathrm{x}}$ Te. J. Appl. Phys. 53, 7099-7101 (1982)

Hurkx, G.A., Klaassen, D.B.M., Knuvers, M.P.G.: A new recombination model for device simulation including tunneling. IEEE Trans. Electron Devices 39(2), 331-338 (1992)

Irvine, J.C.: Recent development in MOCVD of $\mathrm{Hg}_{1-\mathrm{x}} \mathrm{Cd}_{\mathrm{x}} \mathrm{Te}$. In: Proceedings of the SPIE, vol. 1735, pp. 92-99 (1992)

Li, Q., Dutton, R.W.: Numerical small-signal AC modeling of deep-level-trap related frequency-dependent output conductance and capacitance for GaAs MESFET's on semi-insulating substrates. IEEE Trans. Electron Devices 38, 1285-1288 (1991)

Madejczyk, P., Piotrowski, A., Gawron, W., Kłos, K., Rogalski, A., Rutkowski, J.: Morphology issues of $\mathrm{HgCdTe}$ samples grown by MOCVD. In: Proceedings of the SPIE, vol. 7298, pp. 729825-1-729825-10 (2009a)

Madejczyk, P., Piotrowski, A., Kłos, K., Gawron, W., Rogalski, A., Rutkowski, J., Mróz, W.: Surface smoothness improvement of HgCdTe layers grown by MOCVD. Bull. Pol. Acad. Technol. 57, 139-146 (2009b)

Madejczyk, P., Gawron, W., Martyniuk, P., Kębłowski, A., Piotrowski, A., Pawluczyk, J., Pusz, W., Kowalewski, A., Piotrowski, J., Rogalski, A.: MOCVD grown HgCdTe device structure for ambient temperature LWIR detectors. Semicond. Sci. Technol. 28(10), 105017-1-105017-7 (2013)

Martyniuk, P., Gawron, W., Rogalski, A.: Modeling of HOT (111) HgCdTe MWIR detector for fast response operation. Opt. Quantum Electron. 46(10), 1303-1312 (2014)

Maxey, C.D., Ahmed, M.U., Capper, P., Jones, C.L., Gordon, N.T., White, M.: Investigation of parameters to obtain reduced Shockley-Read traps and near radiatively limited lifetimes in MOVPE-grown MCT. J. Mater. Sci. Mater. Electron. 11, 565-568 (2000)

Maxey, C.D., Fitzmaurice, J.C., Lau, H.W., Hipwood, L.G., Shaw, C.S., Jones, C.L., Capper, P.: Current status of large-area MOVPE growth of $\mathrm{HgCdTe}$ device heterostructures for infrared focal plane arrays. J. Electron. Mater. 35, 1275-1282 (2006)

Piotrowski, J., Rogalski, A.: Uncooled long wavelength infrared photon detectors. Infrared Phys. Technol. 46, 115-131 (2004)

Piotrowski, J., Rogalski, A.: High-Operating Temperature Infrared Photodetectors. SPIE Press, Bellingham (2007)

Rogalski, A.: Infrared Detectors. CRC Press, Boca Raton (2011)

Scott, W.: Electron Mobility in $\mathrm{Hg}_{1-\mathrm{x}} \mathrm{Cd}_{\mathrm{x}}$ Te. J. Appl. Phys. 43, 1055-1062 (1972) 